\title{
Augenmensch: Zur Bedeutung des Sehens im Werk Goethes
}

\author{
Vorwort
}

Die hier unter dem Titel „Augenmensch: Zur Bedeutung des Sehens im Werk Goethes“ versammelten Aufsätze stellen die Ergebnisse eines Symposions dar, das vom 7.-9. November 1999 in New York City stattfand. ${ }^{1}$

Ziel des Symposions war es, in Anlehnung an neuere Tendenzen kulturwissenschaftlicher Forschung (Stichwort: „visuality studies“) die sich im Werk Goethes vollziehende Konstruktion des Sehens zu untersuchen. Die Fragestellung hat den Vorteil, dass sie ihrer Intention nach sehr unterschiedliche Werke theoretisch fundiert miteinander zu vergleichen erlaubt und auf diese Weise gattungs- und diskursübergreifende Konstanten erkennen lässt. Gleichzeitig gewährleistet die Interdisziplinarität des kulturwissenschaftlichen Ansatzes eine der Vieldimensionalität des Themas entsprechende Vielfalt der Forschungsperspektiven. So stellen die folgenden acht Beiträge einen Querschnitt durch das Gesamtwerk Goethes dar, der neben bedeutenden dichterischen Texten auch ästhetische, wissenschaftliche und erkenntnistheoretische Arbeiten sowie autobiographische Zeugnisse umfasst. Ist für Goethe der Mensch schlechthin „Zum Sehen geboren, / Zum Schauen bestellt“ (Faust II, 11289-90), so ist das Sehen gleichwohl kein unwandelbarer, in allen Erfahrungsbereichen gleich bleibender Vorgang, sondern etwas höchst Plastisches, das sich bilden und umbilden lässt. Insofern kann man bei Goethe von einem Konzept des Sehens als einer „Kunst“ bzw. „kulturellen Technik“ sprechen. Auf dem in mehreren Beiträgen angesprochenen epistemologischen Feld erreicht diese Technik höchste Dignität, indem sie sich zur „Ideenschau“ läutert, einer visuellen Anschauung ideeller Gehalte. An diesem Begriff, der einerseits eine auf Platon zurückreichende Diskurstradition fortsetzt, andererseits moderne Konzepte der „Übersicht“ bzw. der intuitiven Erfassung von Gesetzmässigkeiten antizipiert, lässt sich die tiefgreifende Transformation semantischer Bestände, die allgemein für das Werk Goethes charakteristisch ist, in aller Deutlichkeit erkennen. Neben der Funktion der Welterschliessung wird in den Beiträgen ebenfalls die eminente Bedeutung betont, die dem Sehen bei Goethe als Medium der Herausbildung von Subjektivität zukommt. Im Blick und erwiderten Blick formieren und stabilisieren sich Muster sowohl intimer als auch öffentlicher Identität. Für dieses „Augenspiel“ (Canetti) entwickelt Goe-

\footnotetext{
${ }^{1}$ Für grosszügige Förderung danken die Herausgeber der Columbia University, dem Deutschen Akademischen Austauschdienst, dem Goethe-Institut, der Johns Hopkins University und der Fritz Thyssen Stiftung.
} 
the eine höchst differenzierte Sprache, die bis in die feinsten Verästelungen der Affektivität hineinreicht. Neben der erkenntnismässigen Relevanz des Sehens und der Bedeutung, die ihm im Werk Goethes bei der Konstitution von Subjektivität zukommt, verbindet die Beiträge schliesslich ein drittes Leitmotiv, das man als die Krisenanfälligkeit des Sehens bezeichnen könnte. Sowohl die Weltzugänge als auch die Identitätsmuster, die sich in Formen des Sehens und des Blickes niederschlagen, sind prekär; sie können sich in einer Proliferation monströser, beängstigender, ins Formlose übergehender Bilder auflösen, können in (Ver-)Blendungen und Blindheiten enden. Dieses Gefährdetsein des Visuellen darf man als eine wichtige Quelle der inneren Dynamik goethischer Texte ansehen. In den einzelnen Studien werden natürlich die erwähnten thematischen Zusammenhänge je nach Gegenstand und Methode der Untersuchung unterschiedlich konkretisiert. Das Gesamtergebnis geht jedoch über die individuellen Befunde hinaus und führt damit vor, wie sich - gerade für die Goetheforschung - Methodenpluralität durch interdisziplinär angelegte Fragestellungen produktiv machen lässt.

Dorothea von Mücke

David E. Wellbery 